\title{
LA OPINION PÚBLICA LATINOAMERICANA FRENTE A LA EDUCACIÓN ${ }^{1}$
}

María Braun (Argentina) ${ }^{2}$

\section{Introducción}

¿Por qué es relevante conocer las opiniones de la población en relación con la educación? La educación es un bien público, una herramienta que contribuye a disminuir las desigualdades sociales, compensando a quienes no han sido privilegiados por su nacimiento, y como tal ha sido siempre altamente valorada por la opinión pública. Como afirma Guillermina Tiramonti en una excelente reflexión sobre el sistema educativo argentino, "la escuela contiene una promesa, muy presente en los sectores populares, de proporcionar los saberes, las habilidades y las titulaciones necesarias para la superación de las limitaciones de origen social". ${ }^{3}$ Evaluar hasta dónde esa promesa es cumplida es relevante, sea para la evaluación de una política pública, sea para su diseño.

¿Por quéutilizar los datos del Latinobarómetro? Encuestas como el Latinobarómetro, que son sistemáticamente aplicadas a muestras representativas de la población, permiten conocer y comparar las actitudes de los latinoamericanos

Los datos que se utilizan en esta nota fueron relevados y procesados por Ana Rapoport. Agradezco a Laura Golbert sus observaciones y comentarios.

2 Socióloga (UBA), Mr en Desarrollo Social (University of London). Socia Directora de MBC MORI y Presidenta de WAPOR Latinoamérica. mariabraun@mbc-mori.com.ar.

3 http://www.lanacion.com.ar/m1/1637687-una-escuela-sin-vocaciontransformadora 
en relación con una serie de temáticas de interés público. En el año 2011 el Latinobarómetro incluyó un set de preguntas específicas relacionadas con cuestiones educativas. ${ }^{4}$ Difundir estos datos, hoy disponibles al público académico, profesional o político, me parece que tiene valor en términos del conocimiento que nos proveen de las sociedades en que vivimos (http://www.latinobarometro.org/latContents.jsp).

¿Cuáles fueron las preguntas que guiaron este trabajo? Básicamente, establecer si existe alguna relación entre el gasto en educación y la opinión de los ciudadanos, detectar las variables que más impactan sobre estas opiniones y sobre las expectativas depositadas en la educación y presentar datos comparados de los diferentes países, particularmente de aquéllos que conforman el Cono Sur. ${ }^{5}$ Cabe destacar, por otro lado, que el interés estuvo puesto fundamentalmente en las evaluaciones relativas a la educación secundaria. Como ha sido señalado por especialistas en el tema, desde los años ochenta, los sistemas educativos de la región enfrentan una situación harto compleja que resulta, entre otras cosas, de una demanda de escolarizar a toda la población durante un período cada vez más largo de la vida. "Si hasta mediados del siglo pasado se trataba de incluir a todos en el nivel primario y sólo a unos pocos en el secundario, hoy se ha establecido la obligatoriedad de la escuela media para toda la población". Es entonces en este estadio de la educación donde encontramos los principales problemas y desafíos en nuestros países.

En 2011 la OEI (Organización de Estados Americanos para la Educación la Ciencia y la Cultura) firmó un convenio con la Corporación Latinobarómetro y la Fundación Carolina con el objetivo de incluir en el Latinobarómetro una serie de preguntas sobre educación. Los resultados de esta encuesta pueden verse en http://www.oei.es/miradas2012.pdf. Más allá de las distintas definiciones al respecto que pudieren haber, estamos tomando como Cono Sur Argentina, Brasil, Chile y Uruguay.

6 http://www.lanacion.com.ar/m1/1637687-una-escuela-sin-vocaciontransformadora 


\section{Algunos datos}

Los años noventa y los años dos mil presenciaron en América Latina una expansión sustancial de la educación secundaria: el gráfico a continuación muestra un aumento de la cantidad de años de escolaridad de la población en todos los países de América Latina, siendo particularmente destacables los casos de Brasil, México y El Salvador

\section{Gráfico 1: Incremento en los años de educación - de 25 a 65 años}

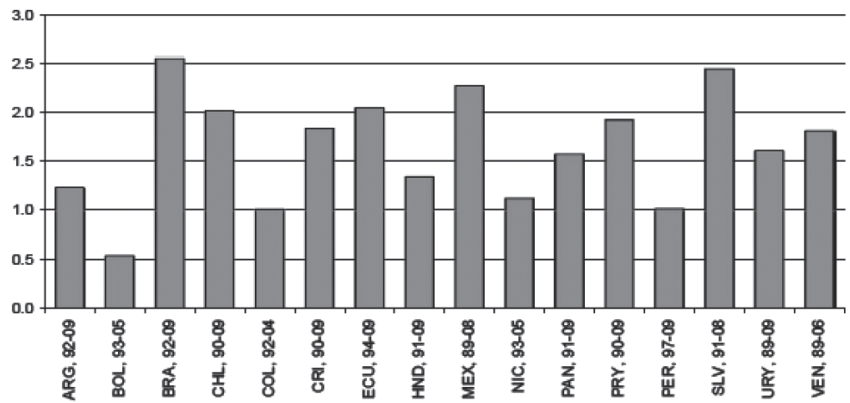

Fuente: Cruces, Garcia Domenech, Gasparini: "Inequality in education evidences for Latin America", Cedlas DT 135, agosto 2012, p. 6

En mayor o en menor medida, en todos los países de América Latina la escuela fue incluyendo nuevas cohortes. Este incremento de la escolarización benefició fundamentalmente a los sectores más bajos de la sociedad, siendo que "el sector público fue el que absorbió a los estudiantes provenientes de los hogares pobres, mientras que las escuelas privadas siguieron atendiendo a los sectores sociales más pudientes". Esto significó un desafío para la escuela media, entre otras cosas porque es precisamente a los 
sectores de menores recursos económicos a quienes les resulta más difícil completar el nivel. ${ }^{7}$

Este proceso de masificación de la educación media se dio con un incremento significativo, en todos los países latinoamericanos, delos recursos aplicados a la educación. Entre 2000y 2010, Argentina, Chile y Brasil duplican el gasto; Uruguay lo triplica. Hoy, la Argentina dedica a la educación el 6,5\% de su PBI (un alto porcentaje si se lo compara con los países de la región y aun con muchos europeos). ${ }^{8}$ Con el recientementelanzamiento del llamado Plan Progresar este porcentaje llegaría al 7\%. ${ }^{9}$

\section{Gráfico 2: Gasto en educación (\% del PIB) por países de América Latina ${ }^{10}$}

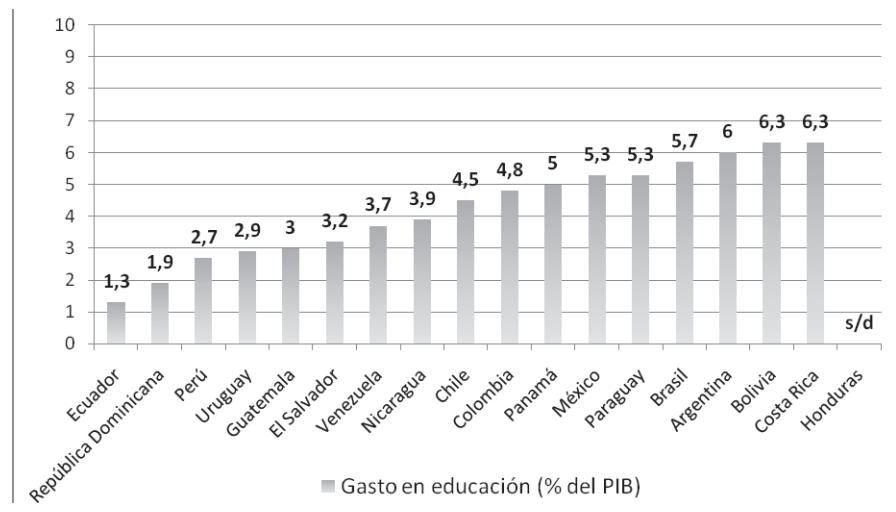

Fuente: HDRO - PNUD

7 Ana Pereyra, "La fragmentación de la oferta educativa en América Latina: la educación pública vs. la educación privada, Perfiles educativos, vol. 30, núm. 120, México, 2008.

${ }_{8}$ Es importante tener en cuenta que una parte muy significativa de este presupuesto se dedica a los salarios docentes.

$9 \quad$ El Plan Progresar, recientemente anunciado por la presidenta Cristina Fernández de Kirchner, que promete ser masivo, es un programa de becas para jóvenes de 18 a 24 que no hayan finalizado estudios secundarios y que sean desocupados o trabajadores informales o incluo formales pero con un salario inferior al mínimo.

10 Los datos de República Dominicana, Ecuador, Nicaragua, Panamá y Paraguay corresponden al año 2000. Los de Bolivia, Guatemala, Uruguay 
El gráfico a continuación muestra cómo fue incrementándose el gasto en educación en las dos últimas décadas en los distintos países de América Latina, siendo los países del Cono Sur -Argentina, Chile, Uruguay y en menor medida Brasil- los que presentan los aumentos más significativos.

Gráfico 3: Gasto público en educación por niño entre 0 y 14 años (en u\$), 1990-2010

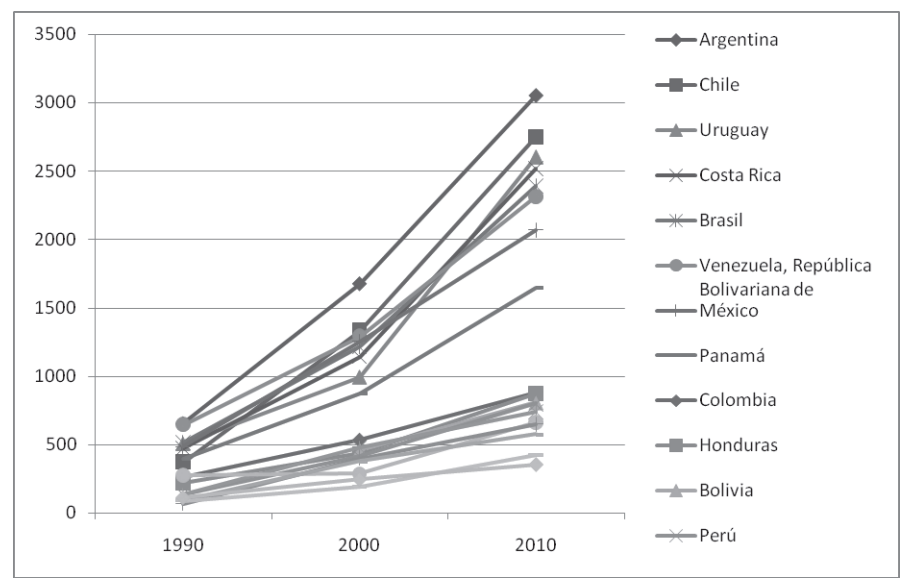

Fuente: Cruces, Garcia Domenech, Gasparini: "Inequality in education evidences for Latin America", Cedlas DT 135, agosto 2012

Ahora bien, este categórico aumento del presupuesto educativo, particularmente en esta última década, así como el esfuerzo del Estado para incorporar a la educación a amplios sectores que antes quedaban afuera, no lograron revertir la situación crítica que vive el sistema educativo en muchos de los países latinoamericanos. Un sistema en el que, como dice un ex ministro de educación refiriéndose a Argentina, la escuela estatal no consigue resolver, ni siquiera en tiempos 
de holgura económica, el problema de la repitencia o el abandono de los estudiantes a lo largo del nivel secundario. ${ }^{11}$

Una muestra de la mala performance de la escuela media en América Latina la ofrecen las pruebas PISA. Hace pocos meses la Organización para la Cooperación y el Desarrollo Económicos (OCDE) difundió los datos de los resultados de las pruebas PISA (Programme for International Student Assessment) correspondientes a 2012, que evaluaron a más de 510000 alumnos de 15 años en matemática, lengua y ciencias. ${ }^{12} \mathrm{El}$ rendimiento en la región fue muy malo: en efecto, los ocho países de la región participantes en este examen (Argentina, Brasil, Chile, Costa Rica, Colombia, México, Perú y Uruguay) calificaron dentro del 25\% de más bajo rendimiento entre los sesenta y cinco países participantes.

En términos algo más específicos, los datos muestran que Chile es el mejor situado al colocarse en el puesto 51. Fue seguido por México en el puesto 53, en el puesto 55 se sitúa Uruguay. Luego sigue Costa Rica, que tampoco consiguió mejorar en relación con la medición anterior -2009- y bajó más de un punto al año situándose en el puesto 56. Brasil se encuentra en el lugar 58 y Argentina quedó en el puesto 59, compartiendo los últimos puestos de la lista con Colombia (62) y Perú en el último puesto. ${ }^{13}$

Los mediocres resultados de las pruebas internacionales y el fenómeno de los alumnos que abandonan las

file:///E:/Mis\%20Documentos/MB/articulos/Latino\%20educacion/ narodosky.htm.

12 Esta prueba se realiza cada tres años desde 2000, y en esta última participaron sesenta y cinco países, es decir, un tercio del total mundial. De ellos, treinta y cuatro son miembros de la OCDE (Organización para la Cooperación y el Desarrollo Económico); los treinta y uno restantes son emergentes, en su mayoría de desarrollo intermedio. De América Latina concursaron la Argentina, Brasil, Chile, Colombia, Costa Rica, México, Perú y Uruguay.

13 Las pruebas PISA mostraron el creciente liderazgo de los países o regiones del Asia Pacífico. 
escuelas estatales para buscar refugio en la educación privada $^{14}$ son sólo algunas de las muestras de que "las políticas empleadas para promover la expansión de la escolarización secundaria en América Latina parecieran estar llegando a los límites de sus posibilidades".15

\section{La opinión pública latinoamericana frente a la educación}

Si las pruebas PISA mostraron el desfasaje existente entre la inversión educativa y los conocimientos alcanzados por los estudiantes de nivel secundario, los datos de opinión pública muestran que esta inversión tampoco se trasladó a una evaluación positiva de la calidad de la educación en su conjunto: sólo un cuarto (el 24,9\%) de los latinoamericanos evalúan positivamente (de 8 a 10 puntos en una escala del 1 al 10) la educación primaria y porcentajes algo más bajos (el 24,4\%) lo hacen en relación con la educación secundaria. Los gráficos a continuación muestran que la mayoría de los latinoamericanos se inclina por valores intermedios (de 4 a 7 puntos), y que en algunos países -Chile, República Dominicana, Perú, Honduras y Guatemala- aproximadamente un cuarto de la población tiene opiniones decididamente negativas (de 1 a 3 puntos).

14 En Argentina la huida de la educación pública es vertiginosa, al punto de que ocho de cada diez nuevos alumnos de primaria y secundaria optaron, en la última década, por establecimientos de gestión privada, http://www.idesa.org/informes/811.

15 Ana Pereyra, "La fragmentación de la oferta educativa en América Latina: la educación pública vs. la educación privada”, op. cit. 


\section{Gráfico 4: Evaluación de la calidad de la educación pública}

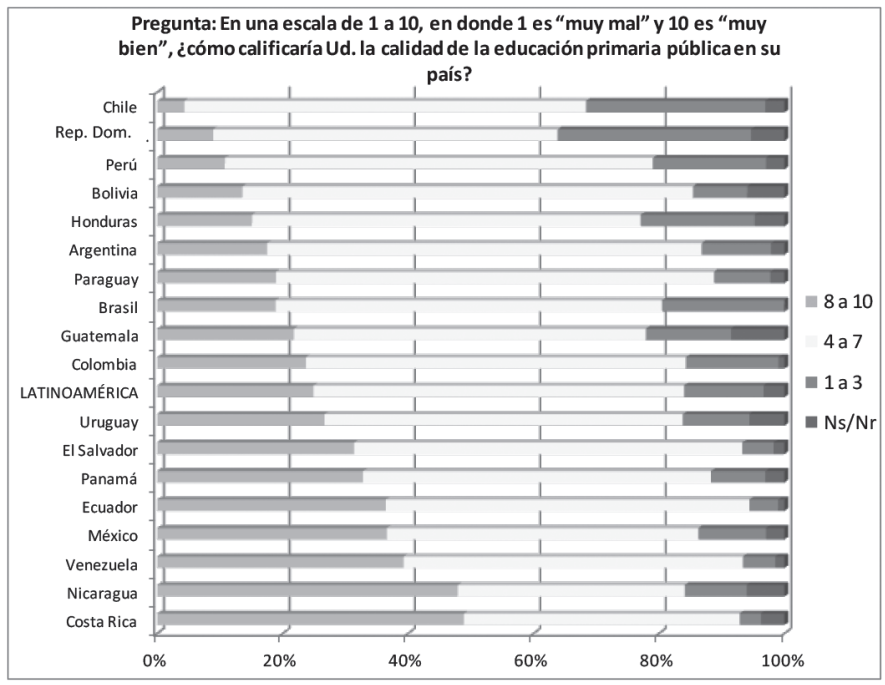

\section{Gráfico 5: Evaluación de la calidad de la educación pública secundaria}

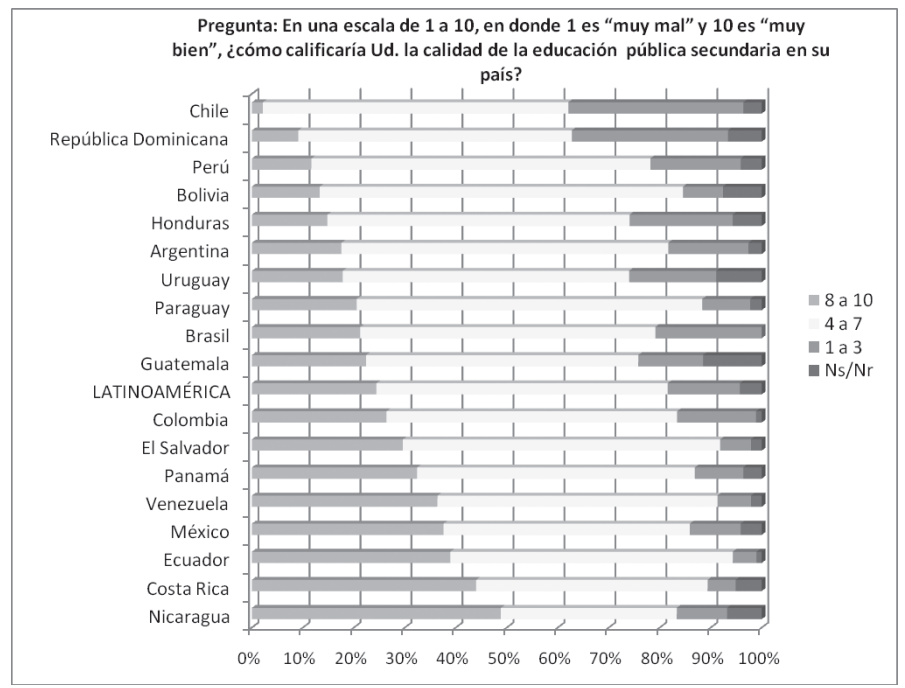


Una primera cuestión a destacar a partir de estos datos hace a las diferencias que presentan los países en relación con estas evaluaciones, Así, si el promedio de la región es relativamente bajo (poco más de un $20 \%$ hace una evaluación positiva de la educación primaria y de la secundaria), en algunos países -Costa Rica, Nicaragua, y en alguna menor medida Ecuador, México y Venezuela- estas evaluaciones superan el $40 \%$, en tanto que en otros estas evaluaciones son significativamente más bajas.

Si tomamos más específicamente los países del Cono Sur -Argentina, Brasil, Chile y Uruguay- que son precisamente aquéllos donde el aumento de la inversión en la última década fue más alta, se observa que:

- Todos estos países se ubican, en términos de la evaluación tanto primaria como secundaria, por debajo de los niveles promedio de América Latina.

- Chile es el país de América Latina que peor evalúa la educación pública, tanto primaria como secundaria.

- Uruguay es el único país donde la evaluación de la educación primaria es significativamente mejor que la de la secundaria. En el resto de los países analizados no se observan diferencias significativas.

- En Brasil la educación secundaria es algo mejor evaluada que la primaria, en tanto que en Argentina no se observa ninguna diferencia. 
Grafico 6: Evaluación positiva de la educación primaria y secundaria en los países del Cono Sur

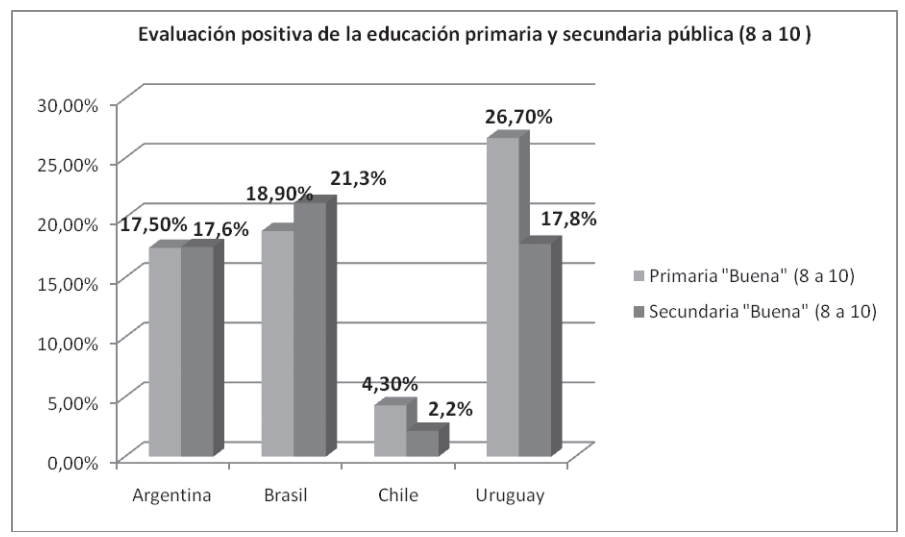

Fuente: Latinobarómetro, medición 2011

Por otro lado, si observamos estos datos para el conjunto de los países latinoamericanos y en función de algunas variables clave, vemos que la evaluación positiva de la educación secundaria es algo más baja entre quienes tienen educación superior y quienes viven en las ciudades capitales, y sólo un poco más alta entre quienes asistieron a una escuela pública 
Gráfico 7: Evaluación de la calidad de la educación secundaria pública según nivel educativo.

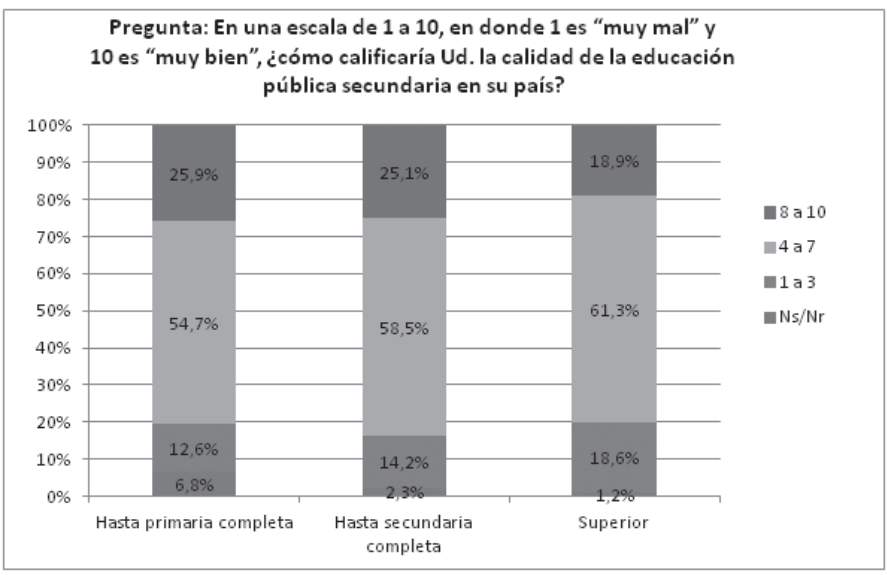

Gráfico 8: Evaluación de la calidad de la educación pública secundaria según tipo de establecimiento (privado o público) al que asistió.

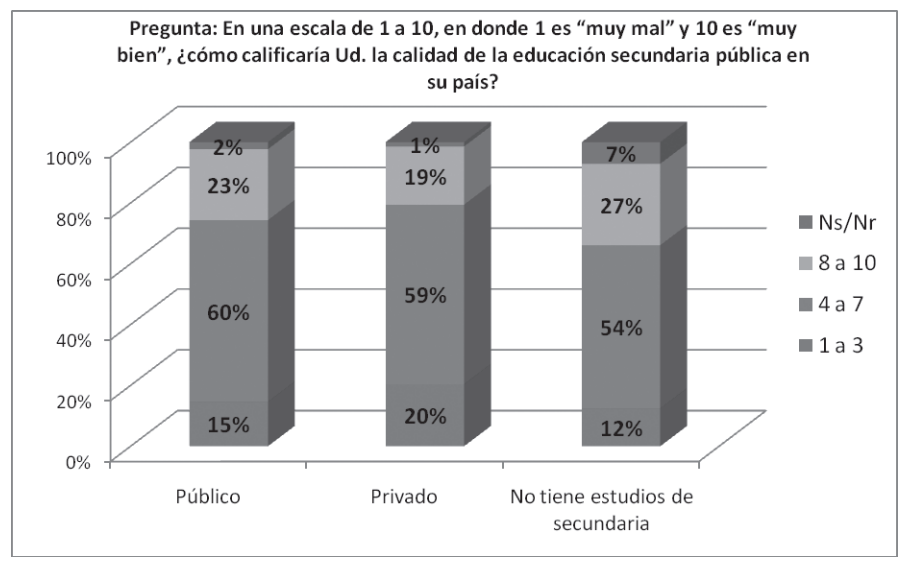


Grafico 9: Evaluación de la calidad de la educación pública secundaria según tamaño de la localidad en que reside.

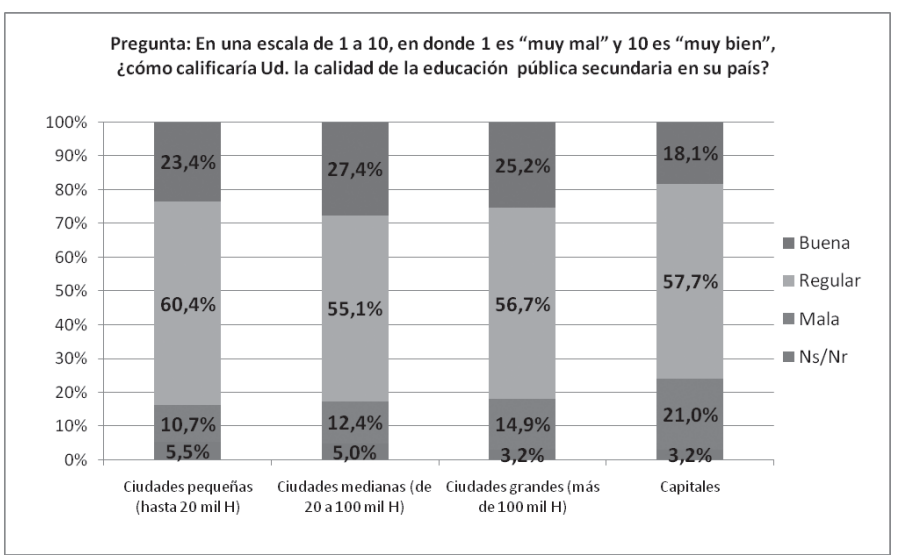

Si nos detenemos en la forma en que los latinoamericanos evalúan los cambios ocurridos en la educación en los últimos años, vemos algunas diferencias significativas. En algunos países -Chile y Argentina manifiestamente, Uruguay en menor medida-, segmentos mayoritarios de la población señalan cambios negativos en la última década; en otros -Costa Rica, Paraguay, Ecuador y Colombia, por ejemplo- son mayoría quienes mencionan cambios positivos. 


\section{Gráfico 10: Evaluación retrospectiva de la educación pública}

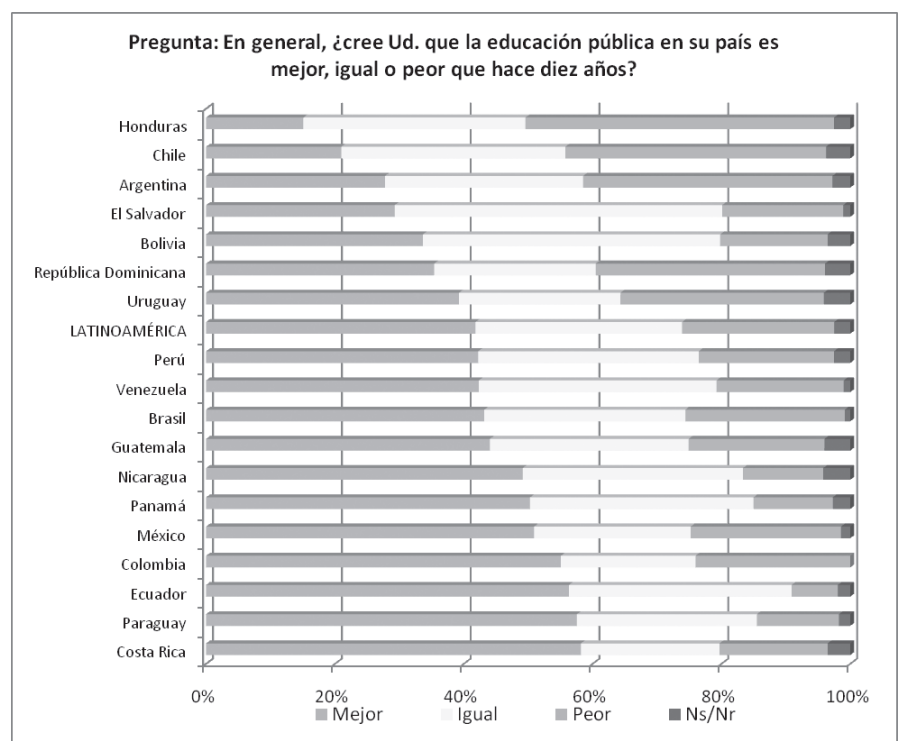

Fuente: Latinobarómetro, medición 2011

Si ponemos el foco en las expectativas, vemos que la mitad de los latinoamericanos son optimistas en relación con una mejora en la educación. En algunos países del Cono Sur-Brasil por ejemplo-, el porcentaje de los ciudadanos que confía en que la educación mejorará en los próximos diez años llega al 60\%, en tanto que en Chile y en Argentina disminuye a poco más del $40 \%$. Cabe señalar que países como Honduras y El Salvador presentan expectativas muy bajas en relación con el futuro. 
Gráfico 11: Expectativas respecto del futuro de la educación pública

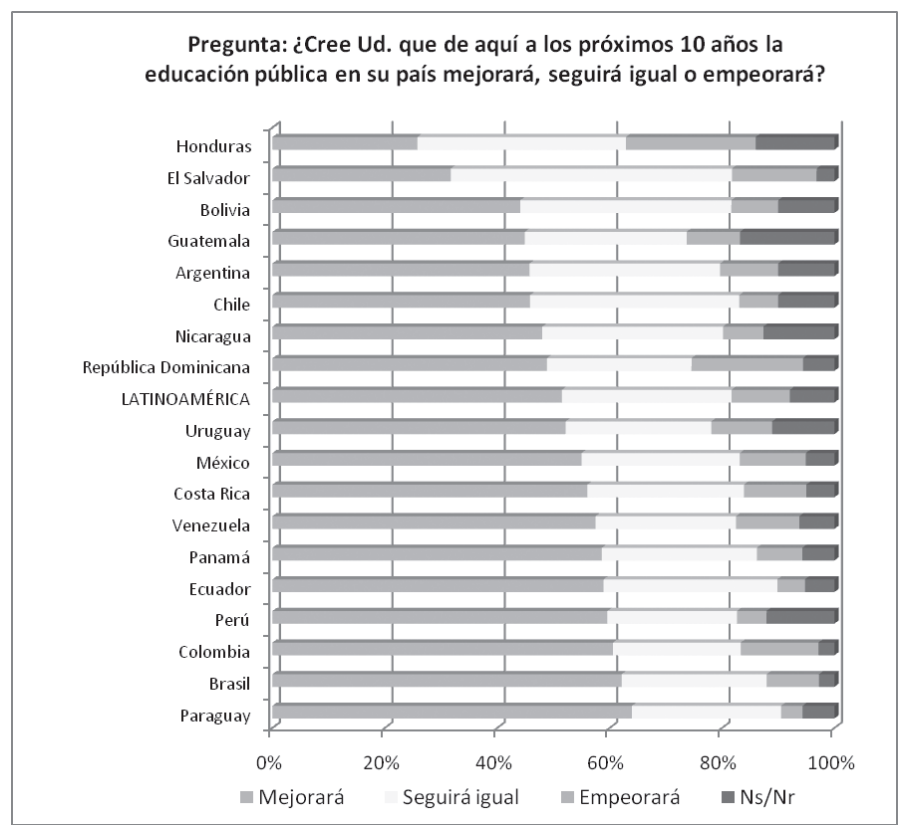

Aunque las diferencias no son demasiado significativas, son los jóvenes quienes más expectativas tienen en relación con el futuro de la educación pública, y los niveles educativos más bajos quienes menos expectativas tienen. 


\section{Gráfico 12: Expectativas respecto del futuro de la educación pública según educación}

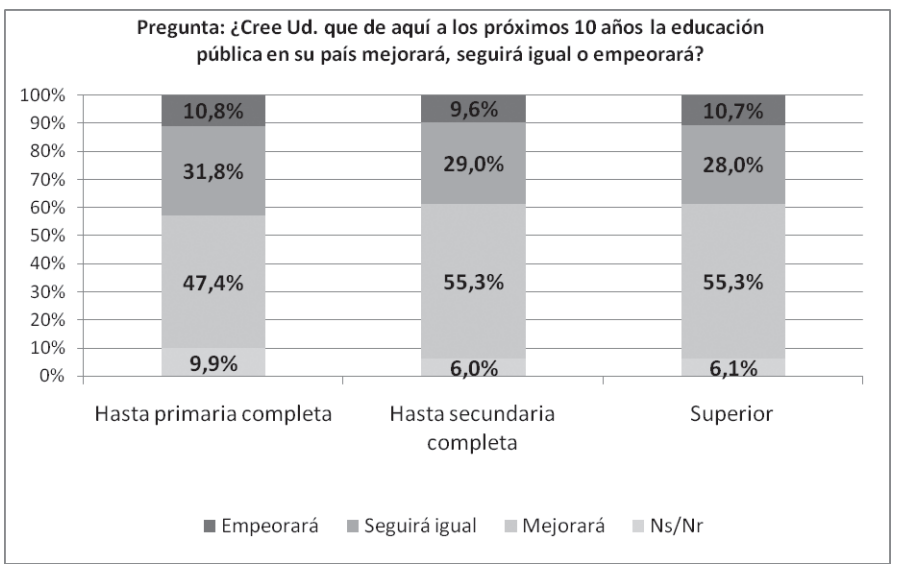

\section{Gráfico 13: Expectativas respecto del futuro de la educación pública según edad}

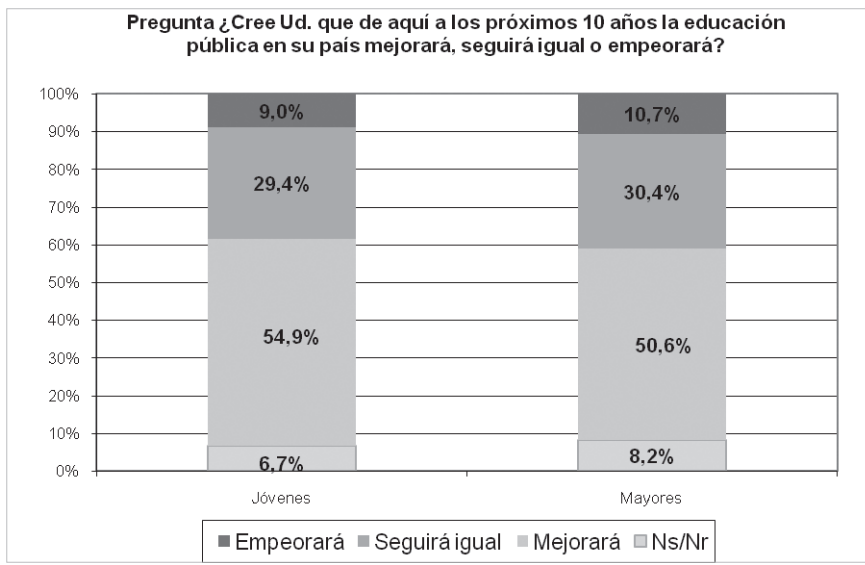


La educación ha sido y pretende ser, lo decíamos al principio, un factor básico de integración de amplios segmentos de la población, particularmente de los sectores desfavorecidos. Ahora bien, no todos los latinoamericanos consideran que este cometido se viene cumpliendo, y cuando se les pide que evalúen la capacidad de sus respectivos sistemas educativos para brindar iguales oportunidades a todos los sectores sociales, las diferencias por país son muy importantes. Cabe señalar que todos los países del Cono Sur, pero particularmente Argentina y Chile, son los países que peor evalúan el desempeño de su sistema educativo en cuanto a su capacidad para ofrecer más oportunidades a todos los sectores sociales

\section{Gráfico 14: Percepción de la educación como factor de integración}

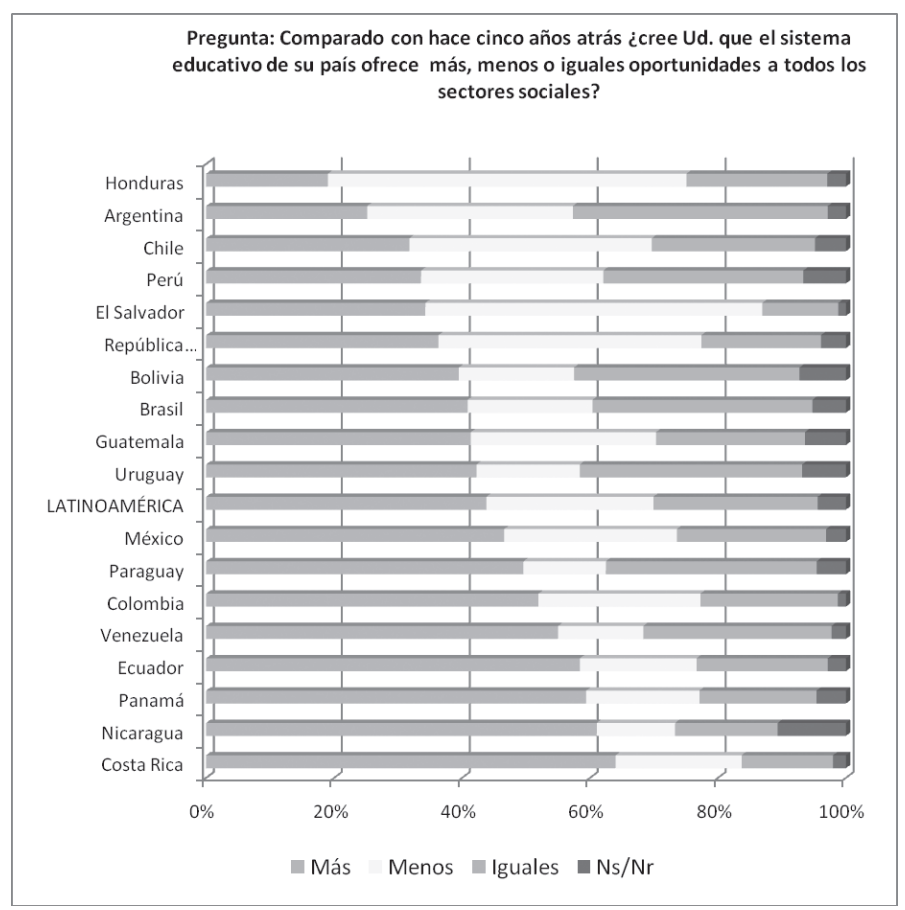


Una mención aparte merece la evaluación que la opinión pública hace de los profesores de las escuelas públicas. Si tomamos los países del Cono Sur vemos que en todas las variables evaluadas -conocimientos, frecuencia en las aulas y capacidad para enseñar- los principales problemas se plantean en Uruguay y en mayor medida aun en Chile. Por otro lado, se observa que la variable más crítica, en todos los casos, es la frecuencia de los profesores en las aulas.

Gráfico 15: Evaluación positiva de las diferentes capacidades de los profesores - Cono Sur

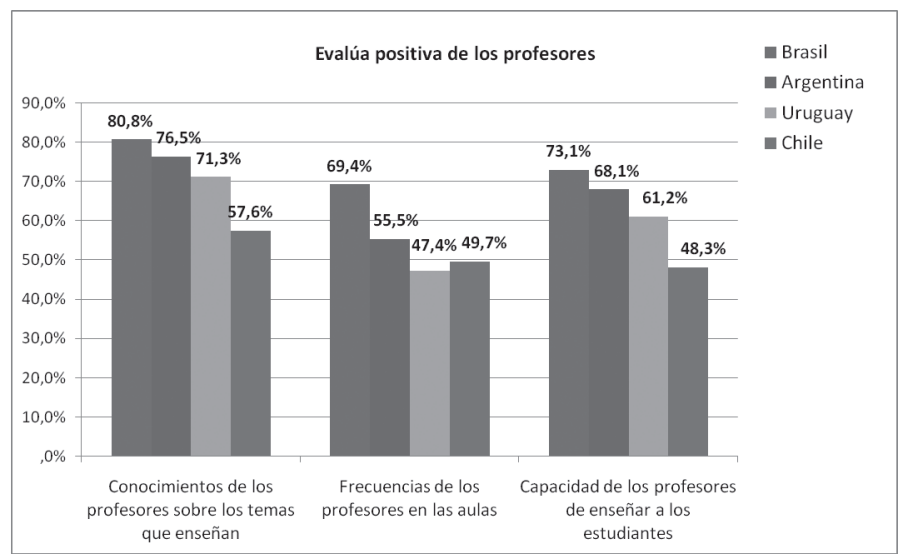

\section{Para terminar}

Aun con las limitaciones que pueda tener el hecho de ser solo uno de los múltiples indicadores que miden el desempeño de una política pública educativa, las pruebas PISA mostraron -y con crudeza, por cierto- que, en el caso de los países de América Latina, la inversión realizada en las dos últimas décadas en educación no se tradujo en un 
mejoramiento en la performance de los estudiantes secundarios. Lo visto a partir de estos datos del Latinobarómetro nos permite afirmar que este gasto tampoco impactó sobre la opinión pública: la opinión pública latinoamericana, particularmente en algunos de los países, es bastante crítica respecto de la educación y, si bien las expectativas depositadas en la misma son altas, la confianza en que en los próximos años la educación pública vaya a mejorar es realmente limitada.

Por otro lado, los datos muestran que las grandes diferencias son entre los países y no según los diferentes segmentos sociales. Así, variables "duras", como la edad de los entrevistados, la educación, el tipo de establecimiento donde realizó sus estudios o el tamaño de la localidad donde habita, casi no impactan sobre las opiniones sobre la educación. Es que la educación es, efectivamente, un bien "universal", una demanda que incluye a todos los sectores sociales, una expectativa de que se puede ser mejor y vivir en una sociedad mejor. La forma en que esas demandas y expectativas se expresan está fuertemente determinada, seguramente, por la historia político institucional de cada país, por sus tradiciones e inclusos sus mitos. 